\title{
Longitudinal relationships between lifestyle and cardiovascular and bone health status indicators in males and females between 13 and 27 years of age; a review of findings from the Amsterdam Growth and Health Longitudinal Study
}

\author{
W. van Mechelen ${ }^{1,2, *}$, J.W.R. Twisk ${ }^{1}$, H.C.G. Kemper ${ }^{1}$, J. Snel ${ }^{3}$ and G.B. Post ${ }^{1}$ \\ 'Institute for Research in Extramural Medicine, Faculty of Medicine, Vrije Universiteit Amsterdam, \\ NL-1081 BT Amsterdam, the Netherlands \\ ${ }^{2}$ Department of Social Medicine, Faculty of Medicine, Vrije Universiteit Amsterdam, NL-1081 BT Amsterdam, \\ the Netherlands \\ ${ }^{3}$ Department of Psychophysiology, Faculty of Psychology, University of Amsterdam, NL-1018 WB Amsterdam
}

Accepted: 16 July 1999

\begin{abstract}
The Amsterdam Growth and Health Longitudinal Study is longitudinal co-hort study on 181 males and females initially aged 13 years, with follow-up measurements at ages 14, 15, 16, 21 and 27 years.

Methods: Anthropometrical, biological and lifestyle parameters, and age 27 also bone mineral density (BMD) of the lumbar spine (L2-L4), were measured repeatedly. Adolescent dietary intake and physical activity was related to adult cardiovascular and bone health status indicators by MLR; longitudinal relationships between physical activity and dietary intake, and cardiovascular health status indicators were assessed by GEE-analysis.

Results: Adolescent physical activity was not related to most of the indicators of adult cardiovascular health status, with the exception of a positive relationship with the waist-to-hip in females; in males 'energetic' adolescent physical activity contributed significantly to adult BMD; both in males and females, when taking the entire longitudinal period into account, peak strain physical activity was a relatively more important predictor of adult BMD than 'energetic' physical activity; calcium intake during adolescence was not a significant predictor of bone health status measured at age 27 , both in males and females; a consistent significant positive longitudinal relationship was found between physical activity and serum HDL-cholesterol and significant negative longitudinal relationships were found with the subscapular skinfold and with body fat mass; a positive longitudinal relationship was found between serum total cholesterol (TC) and cholesterol intake, saturated fat (SFA) intake and the Keys-score; a negative longitudinal relationship was found between TC and polyunsaturated fat and total energy intake; for HDL a positive longitudinal relationship was found with SFA intake; low tracking was found for physical (in-) activity and dietary intake variables.
\end{abstract}

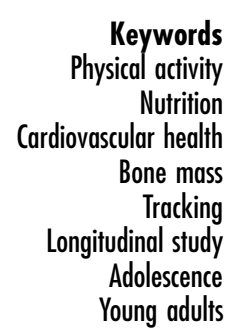

\section{Key Messages}

1. Physical activity will only have a beneficial influence on cardiovascular health status if it is of a sustained nature. Once the physical activity stops the beneficial influence on cardiovascular health will disappear.

2. In modern western society adolescent physical activity is of more importance to adult bone health, than calcium intake during adolescence.

3. Physical activity behaviour is no constant over time, nor is physical inactivity behaviour. Therefore the entire population is the target group for public health interventions that aim at the improvement of levels of health enhancing physical activity.

\section{Introduction}

In modern western society nowadays the majority of morbidity and mortality results from multifactorial chronic diseases. Examples of such chronic diseases are cardiovascular disease, osteoporosis, non-insulin dependent diabetes mellitus, certain forms of cancer, 
etc. Next to genetic predisposition, environmental and lifestyle factors, such as unfavourable nutrition and physical inactivity, play an important role in the pathogenesis of these diseases. The role of lifestyle factors in the aetiology of disease can be either direct (independent) or indirect (dependent). For instance, it is known that there is an independent relationship between physical inactivity and an increased risk for cardiovascular disease mortality ${ }^{1}$. However, it is also known that physical inactivity can lead to an increase in body weight ${ }^{2}$, which in turn is a risk factor for cardiovascular disease $^{3}$. The picture becomes even more complex if one realizes that body weight reflects energy balance in which both energy intake (nutrition) and energy output (physical activity) play an important role ${ }^{2}$ and that nutritional factors are also independently related to cardiovascular disease risk ${ }^{4}$.

Most of the scientific information in humans on the complex inter-relationships between lifestyle factors and chronic disease stems from observational or experimental epidemiological studies in adult populations. However, it is well accepted that the origin of chronic disease lies in part in childhood lifestyle ${ }^{5}$. Given this fact, it is of great importance that prevention of chronic diseases starts as early in life as possible, by advocating a healthy lifestyle. In order to set out effective preventive strategies already from childhood onwards it is necessary to have knowledge of the (inter-)relationships between childhood lifestyle and adult health. The questions asked here are

(1) the extent to which childhood lifestyle actually relates to adult health and

(2) the extent to which changes over time in lifestyle actually relate to changes in health status.

These questions can only be answered using information derived from longitudinal studies. Such a study must have repeatedly measured both lifestyle and health status indicators already from an early age onwards into adulthood. To our knowledge only one such longitudinal study exists that has measured both lifestyle (nutrition, physical activity, smoking and alcohol intake) and health status indicators (in particular for cardiovascular disease and for osteoporosis), i.e the Amsterdam Growth and Health Longitudinal Study (AGHLS).

A number of papers from the AGHLS have been published concerning the two questions mentioned above. The purpose of this paper is to review and summarize the most important findings of these AGHLS papers, in particular regarding the longitudinal relationship between nutrition and physical activity lifestyle factors on the one hand and biological health status indicators for cardiovascular disease and bone mineral density on the other hand.

\section{Subjects and design}

The Amsterdam Growth and Health Longitudinal Study is a longitudinal study that started in 1977. During the first four years of the study four consecutive measurements were carried out on 233 subjects. In 1985 the study continued with 200 subjects and in 1991 a sixth measurement was carried out on 181 subjects. The initial age of the subjects was $13.1( \pm 0.8)$ years and after fifteen years of follow-up, at the measurement in 1991, the age of the subjects was $27.1( \pm 0.8)$ years. The population used in the studies reviewed, consists of the 181 subjects ( 83 males and 98 females) who remained in the study up to the measurement at the age of 27 years. The total amount of missing observations during the whole period of measurement for these 181 subjects was about 2 percent ${ }^{6}$.

The initial goal of the Amsterdam Growth and Health Study was to describe the natural development of growth, health and lifestyle of a group of healthy adolescent boys and girls. Because of the long duration of the study it became possible to investigate longitudinal relationships between lifestyle parameters and health status indicators.

At each year of measurement anthropometrical parameters (body height, body weight, body composition), biological parameters (serum lipoprotein levels, blood pressure, physical fitness) and lifestyle parameters (nutritional habits, smoking behaviour, daily physical activity) were measured. At age 27 , also bone mineral density of the lumbar spine (L2-L4) was measured.

The aims of all the analyses described in the papers that are reviewed here, were:

(1) to relate health status measured at adult age (i.e. at the age of 27 years) to lifestyle parameters measured during adolescence (i.e. at age 13-16 years) (see Fig. 1) and

(2) to analyse the longitudinal relationship between a lifestyle predictor variable and a health status indicator outcome variable (see Fig. 2).

\section{Methods}

\section{Lifestyle}

Dietary intake was measured by a modification of the cross-check dietary history. In this dietary history, which was specially developed for the Amsterdam Growth and Health Longitudinal Study ${ }^{7}$, all subjects were asked to recall their usual dietary intake by reporting frequency, amounts and methods of preparations of the foods consumed. The method was used to assess the usual food intake during the previous month. All consumed food items were transformed into nutrients by the Dutch Food Composition Table ${ }^{8}$. For 


\section{Lifestyle parameters}

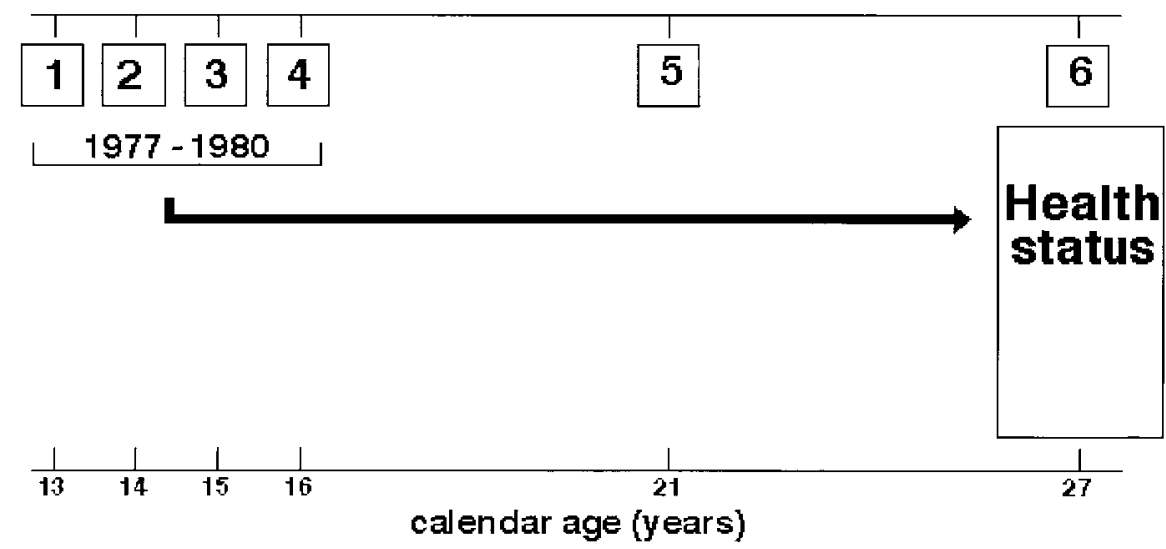

Fig. 1 Relationship between health status measured at adult age (i.e. at the age of 27 years) and lifestyle parameters measured during adolescence (i.e. at age 13-16 years)

the analyses reviewed here the daily intake of the following nutrients was calculated: (1) the intake of saturated fat (SFA), expressed as energy percentage; (2) the intake of poly unsaturated fat (PUFA), expressed as energy percentage; (3) the ratio between the absolute intakes of PUFA and SFA (P:S ratio); (4) the intake of carbohydrates, expressed as energy percentage and; (5) the intake of cholesterol (Chol), expressed in $\mathrm{mg} / \mathrm{mJ}$.

With the dietary history interview also alcohol consumption (expressed in gram per week) and smoking behaviour, i.e. the amount of tobacco (in g) smoked per week, were measured. Daily physical activity was measured by a structured interview, which was developed for the Amsterdam Growth and Health Longitudinal Study ${ }^{9}$. With this interview the total time spent on physical activities in relation to school and work, and on other activities (e.g. organized sports activities, unorganized sports activities, other leisure time activities etc.) was measured. The measured times were combined with the intensity of the different activities to calculate a total weighted (energetic) activity score (expressed in METs/week; 1 MET equals energy expenditure whilst sitting at rest). The activity interview covered a period of the previous three months. Both the cross-check dietary history and the daily physical activity interview were shown to be quite valid measurements of dietary intake and daily physical activity $^{7,9}$.

\section{Biological bealth status indicators for cardiovascular disease}

Blood pressure (BP) was measured with an indirect method. A standard pressure cuff was placed around

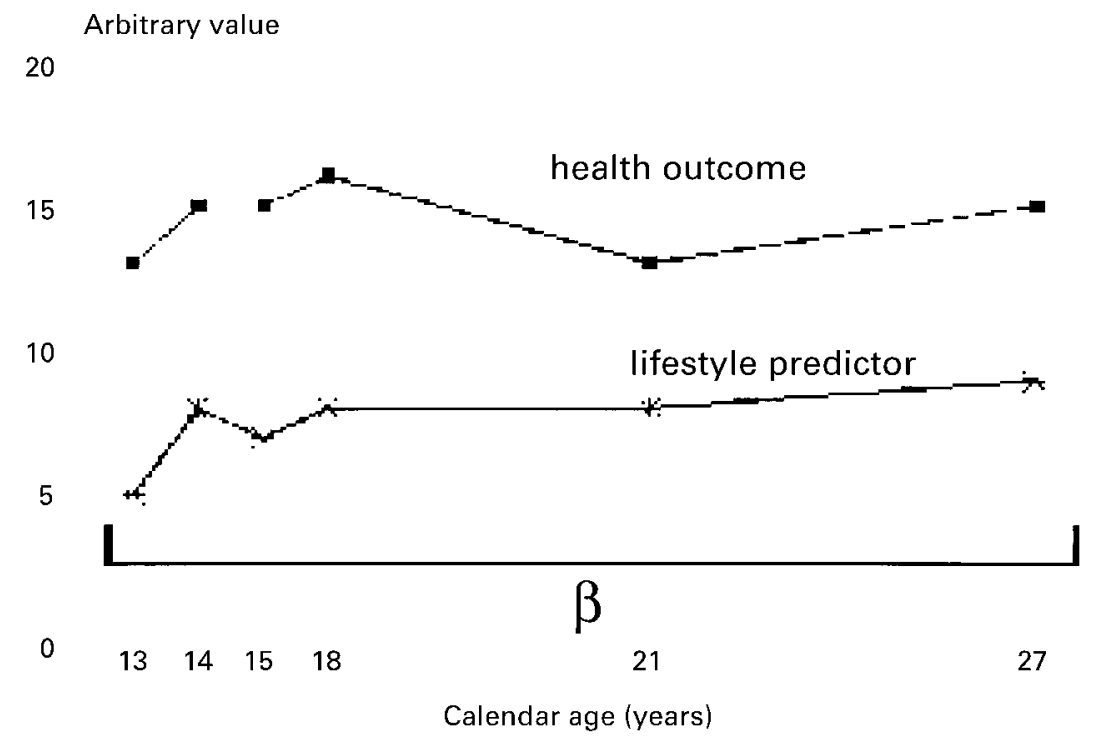

Fig. 2 Longitudinal relationship between a lifestyle predictor variable and a health status indicator outcome variable 
the left upper arm. With a sphygmomanometer diastolic blood pressure (DBP) and systolic blood pressure (SBP) were measured twice and the lowest value was recorded. For the determination of serum lipoprotein concentrations approximately $10 \mathrm{ml}$ of venous blood was taken from the forearm vein with subjects in a non-fasting state. The concentration of total serum cholesterol (TC) was analysed according the methods of Huang et al. ${ }^{10}$ and Abell et al. ${ }^{11}$ and the concentration of high density lipoprotein cholesterol (HDL) was analysed according to Burstein and Samaille $^{12}$. The TC/HDL ratio was derived from the above measurements. Body fatness was operationalized as the sum of four skinfolds (SSF); i.e. biceps, triceps, subscapular and suprailiac. Skinfold thickness was measured with a Harpenden skinfold caliper and expressed in $\mathrm{mm}^{13}$. Finally, cardiopulmonary fitness was defined as the maximum oxygen uptake $\left(\mathrm{VO}_{2^{-}}\right.$ max). $\mathrm{VO}_{2}$-max was measured by a graded treadmill test until exhaustion ${ }^{14}$ and expressed in $\mathrm{ml} \mathrm{min}^{-1} \mathrm{~kg}^{-2 / 3}$.

\section{Bone mineral density measured at age 27}

Bone mineral density (BMD) of the lumbar spine (L2L4) was measured by dual X-ray absorptiometry (DXA; Norland XR-26) at the Department of Nuclear Medicine of The Vrije Universiteit Hospital. The coefficient of variation of DXA for repeated measurements of the lumbar BMD in human subjects at the department is $1.3 \%$ for short-term reproducibility ( $24 \mathrm{~h}$ ) and $2.3 \%$ for long-term reproducibility (2-6 months).

\section{Data analysis}

To evaluate the relationship between lifestyle measured during adolescence and health status indicators at age 27 multiple linear regression (MLR) analyses were carried out. All analyses were carried out correcting for sex and also correcting for the 'other' lifestyle factors (e.g. when the relationship between adolescent nutritional factors and a cardiovascular health status indicator was calculated, the analyses was carried out correcting for sex, smoking, alcohol intake and physical activity). For each analysis the interaction between the lifestyle factor concerned and sex was added to the model. If a significant interaction was found the standardized regression coefficient was calculated for each sex separately. For each analysis the significance level was set at $p<0.05$. All analyses were carried out by SPSS/PC.

The longitudinal relationships between a lifestyle factor and a health status indicator was analysed with generalized estimating equations (GEE) ${ }^{15}$. With GEE the longitudinal relationships between a lifestyle factor and a health status indicator was analysed using all available data, under correction of time-independent (i.e. sex) co-variates, time and time-dependent 'other' lifestyle factors. The advantage of this method is that it takes into account that the repeated observations of each individual are not independent. Using GEE with continuous outcome variables is comparable to a (longitudinal) linear regression analysis. As a result standardized regression coefficients were calculated, which indicate the independent longitudinal relationships between a predictor (lifestyle) variable and an outcome (health status indicator) variable along the whole longitudinal period. The magnitude of the coefficients ranges between 0 and 1 , which makes the coefficient interpretable as a longitudinal (partial) correlation coefficient. After assessing the main effects also the interactions between the predictor variable and sex, and between the predictor variable and time were analysed. The interaction terms were formed by a multiplication of the two parameters involved. Here also in all analyses a $5 \%$ significance level was used. All GEE analyses were carried out with the Statistical Package for Interactive Data Analysis ${ }^{16}$. All longitudinal GEE results presented concern the outcome of multivariate analyses, in which a correction is made for all 'other' lifestyles mentioned above, age and sex (e.g. when the longitudinal relationship between physical activity and HDL is analyses, this relationship is corrected for nutrition, smoking, alcohol intake, age and sex).

\section{Results}

\section{Adolescent physical activity and adult cardiovascular bealth status indicators}

Twisk et $a l^{17}$ analysed the relationship between adolescent physical activity and the following cardiovascular health status indicators measured at the age of 27 years: serum lipoprotein levels (TC and HDL), blood pressure (DBP and SBP), body fatness (SSF) and body fat distribution (waist-to-hip ratio [WHR]). These analyses were corrected for nutritional factors (energy percent from fat intake, energy percent from protein intake and energy percent from carbohydrate intake), smoking and alcohol intake; the latter factor being of only minor significance because of the low alcohol intake during adolescence (average 0.2 and 0.3 gram/ week for the males and females respectively). It was found that adolescent physical activity was not related to most of the adult cardiovascular health status indicators, with the only exception of a positive relationship with the WHR in females: $\beta=0.51 \quad(p<0.01)$. This picture changed only slightly when the physical activity data covering the entire longitudinal period (i.e. all data collected between 13 and 27 years of age) were used to predict adult cardiovascular health. Then the following significant relationships were found between physical activity and 1 . SSF: $\beta=-0.20(p<0.01$, both in males and females); 2 . WHR in females: $\beta=0.26(p<0.01)$.

These findings were more or less confirmed in a comparable study by Van Lenthe et al. ${ }^{18}$. They related 
the physical activity data collected over the entire longitudinal period with indicators of a central pattern of body fat measured at age 27 , i.e. the subscapular skinfold thickness and the waist circumference. They found in males, after adjustment for the SSF, a positive relationship of physical activity with the subscapular skinfold $(\beta=0.15 ; p<0.01)$, explaining $2.3 \%$ of its variance.

\section{Longitudinal relationships between physical activity and cardiovascular bealth status indicators}

Twisk et al. ${ }^{19}$ analysed the longitudinal relationship between physical activity and TC, HDL and the TC/HDL ratio, correcting for body fatness, lean body mass and maximal oxygen uptake. The only significant longitudinal relationship found was that between physical activity and HDL: $(\beta=0.05 ; p<0.05)$.

In another study Twisk et al. ${ }^{20}$ looked at the longitudinal relationship between physical activity and indicators of body fatness (i.e. BMI and SSF). They found in this analysis that physical activity was negatively related to SSF: $(\beta=-0.06 ; p<0.01)$. No longitudinal relationship was found with BMI.

In the earlier mentioned study Van Lenthe et al. ${ }^{18}$ also looked at the longitudinal relationship between physical activity and the subscapular skinfold. They found that the change in physical activity was negatively related to the change in the subscapular skinfold $(\beta=-0.10 ; p<0.001)$. This analyses was adjusted for the SSF.

The study of Kemper et al. ${ }^{21}$ is the third study that was carried out along these lines. They investigated the longitudinal relationship between the development of fat mass and the development of physical activity. In order to do so, a slightly different approach was used than described in the methods section: in short at each longitudinal measurement the subjects were divided into two groups with respect to their fat mass using threshold values for percentage body fat ( $>20 \%$ for males and $>30 \%$ for females) and BMI $\left(>25 \mathrm{~kg} \cdot \mathrm{m}^{-2}\right)$. Applying the GEE-method for both outcome variables Odds Ratios (OR) were calculated comparing the groups below (reference) and above the threshold value. These analyses gave a significant $O R$ of 0.81 (95\% confidence limits: 0.69-0.96) for body fat mass, indicating that a relatively high level of physical activity was related with a body fat mass below the threshold value. No relationship was observed for BMI.

Finally the results of the study of Van Mechelen et $a{ }^{22}$ should be cited here. In epidemiological studies resting heart rate is sometimes used as a proxy measure of physical activity ${ }^{22}$. In their study Van Mechelen et al $^{22}$ studied the longitudinal relationship between resting heart rate and biological health status factors for cardiovasular disease (i.e. DBP and SBP, TC and
HDL, SSF and cardiopulmonary fitness), adjusting for smoking. They found that resting heart rate was significantly associated with DBP $(\beta=0.15 ; p<0.001)$, SBP $(\beta=0.14 ; p<0.001)$, SSF $(\beta=0.05 ; p<0.05)$ and maximal oxygen uptake $(\beta=-0.10 ; p<0.001)$. The association with SSF disappeared when, in addition to the correction for smoking, a correction for habitual physical activity was added to the equation.

\section{Longitudinal relationships between dietary patterns and cardiovascular health status indicators}

Twisk et al. ${ }^{19}$ looked in a multivariate longitudinal GEEanalysis at the relationship between the daily intake of SFA, PUFA, the ratio between PUFA and SFA (P:S ratio), the daily intake of carbohydrates and the daily intake of cholesterol on the one hand and TC, HDL, and TC/ HDL ratio on the other hand. They also looked at the longitudinal relationship between the Keys-score and the biological health status indicators for cardiovasular disease mentioned above. Regarding the relation between dietary intake and lipoprotein levels often this Keys-score is used ${ }^{23}$. In the Keys-score the energy percentage of the intake of SFA, the energy percentage of the intake of PUFA and the intake of cholesterol are combined into one weighted score. The Keys-score is calculated by $1.35 \times(2 \times$ SFA - PUFA $)+1.5 \times Z$, where $Z$ is the square root of the dietary intake of cholesterol expressed in mg/1000 Kcal. The results of the multivariate GEE-analysis revealed that the longitudinal development of TC over a period of 15 years (from 13 to 27 years of age) was inversely influenced by the longitudinal development of the P:S ratio $(\beta=-0.07$ : $p<0.01$ ) and positively by the development of the cholesterol intake $(\beta=0.08: p<0.01)$. When the development of the P:S ratio was substituted by the development of the actual intake of PUFA and the development of the actual intake of SFA as two separate variables, both were significantly related to the development of TC. For the intake of PUFA a $\beta=-0.06(p<$ $0.01)$ and for SFA a $\beta=0.05(p<0.05)$ was found. When the Keys-score was used as predictor variable instead of the intake of SFA, PUFA and cholesterol separately, results showed a significant positive relation with the development of TC $(\beta=0.13 ; p<0.01)$. No significant relationships were found between the longitudinal development of TC and the development of the intake of carbohydrates. No significant relations were found between the longitudinal development of HDL and the development of all dietary parameters. The longitudinal development of the TC/HDL ratio was positively related to the development of the intake of carbohydrates $(\beta=0.06 ; p<0.05)$. The development of all other dietary parameters was not significantly related to the longitudinal development of the TC/ HDL ratio. 
The longitudinal relationships between dietary patterns (i.e. intake of total energy [EN], animal protein, SFA, PUFA and Chol) and cardiovascular health status indicators (SSF, DBP and SBP, TC and HDL) were described also by Post et $a .^{4}{ }^{4}$. After multivariate analyses they found significant longitudinal associations between TC and EN $(\beta=-0.10 ; p<0.01)$;. SFA $(\beta=0.06$; $p<0.05)$; PUFA $(\beta=-0.05 ; p<0.05)$ and; Chol $(\beta=0.06$; $p<0.05)$. They also found significant longitudinal associations between HDL and SFA $(\beta=0.07 ; p<0.05)$. These associations were similar to the ones found in the Twisk et al. ${ }^{19}$ study.

\section{Adolescent physical activity and calcium intake, and adult bone mass density}

In 1991 bone mass density (BMD) of the lumbar spine (L2-L4) was measured in the AGHLS. The outcome of this measurement was with multiple linear regression analysis related to adolescent calcium intake divided by body height (CAIH) and weight bearing physical activities $^{24}$. Since BMD is also determined by body weight, this variable was also entered into the analysis. The analyses were carried out for males and females separately. For males a highly significant relationship was found between adolescent physical activity and BMD measured at age 27 years, with $\beta=0.18(p<0.001)$ and an explained variance of $16 \%$. When the entire period (i.e. from 13-27 years) of exposure to physical activity was taken into consideration the explained variance increased only slightly to $17 \%$. For females adolescent body weight was the only variable found to be a significant predictor of adult BMD: $\beta=0.56 ; p<$ 0.01. In this study, in contrast to the expectation adolescent calcium intake was not a predictor of adult BMD.

Point of critique to the Welten et al. ${ }^{24}$ study was the fact that the weight bearing activities were expressed in a metabolic unit (METs/week). These weight bearing activities can be classified as 'energetic' weight bearing activities. However, in relation to BMD it is mechanical strain of the weight bearing activities that is of paramount importance and not the energy expenditure resulting from these activities. Therefore Groothausen et $a l .{ }^{25}$ re-classified the physical activities in three different categories of 'peak strain physical activity' (PSPA) according to literature values for ground reaction forces, regardless of the metabolic cost of activities. They then studied by multiple linear regression analysis the relationship between adolescent peak strain physical activity (PSPA) and BMD of the lumbar spine measured at age 27 . The analysis was corrected for body weight and sex. Calcium intake was not taken into consideration given the outcome of the Welten et $a .^{24}$ study. The outcome of this analysis showed that both body weight and PSPA measured at age $13,14,15$ and 16 years were significantly related to lumbar BMD measured at age 27, whereas sex was not. For PSPA the following regression coefficients were found for the ages 13, 14, 15 and 16 years respectively: $\beta=0.18(p<0.05), \beta=0.17(p<0.05), \beta=0.09$ (n.s.) and $\beta=0.16(p<0.05)$, explaining $7,8,6$ and 11 percent of the variance of lumbar BMD. When again exposure to PSPA over the entire period was taken into consideration this explained variance increased to $24 \%$.

\section{Discussion}

The purpose of this paper was to review recent findings of the Amsterdam Growth and Health Longitudinal Study on the relationship between adolescent physical activity and nutrition, and adult health status indicators (i.e. indicators for cardiovascular and bone health).

From the studies reviewed it can be concluded that adolescent physical activity was not related to most of the indicators of adult cardiovascular health status, with the exception of a positive relationship with the WHR in females. When trying to explain this lack of effect of adolescent physical activity on adult cardiovascular health status indicators one should take some basic exercise physiological principles into account. It has been known for a long time that regular exercise leads to a more favourable cardiovascular disease risk profile in terms of lowering levels of biological risk factors ${ }^{26}$. However the mode of physical activity that brings about these favourable effects is of an endurance type training, performed regularly (3 times a week, $20 \mathrm{~min}$ ), at a level of intensity of at least $50-80 \%$ of maximum oxygen uptake ${ }^{27}$. It is also known that this kind of regular exercise should be sustained in order to maintain these favourable levels of effect. Perhaps the mode of physical activity performed by the subjects in our study during adolescence did not meet the physical activity quality criterion mentioned above. Consequently it may have been unlikely that the exerted levels of physical activity would have had the potential to bring about a favourable effect anyway. However, the issue of sustained physical activity over longer periods of time needed to induce permanent effects provides a better explanation for our lack of findings. When discussing sustained physical activity one should look at the phenomenon of tracking. Tracking can be described as the stability of a certain lifestyle or health status indicator over time, within a certain individual. Twisk et al. ${ }^{28}$ calculated longitudinal tracking coefficients for the variables relevant to this review. They found a low but significant tracking coefficient for physical activity over the 15 year period: $\beta=0.34(p<$ 0.05 ). This lack of tracking of physical activity in the AGHLS was also described by Van Mechelen and Kemper $^{29}$. The lack of tracking of physical activity is a general phenomenon recently confirmed by Malina in a review study ${ }^{30}$. Twisk et al. ${ }^{28}$ also calculated significant 
$(p<0.05)$ tracking coefficients for the intake of fat $(\beta=$ $0.42)$, the P:S ratio $(\beta=0.33)$, the intake of carbohydrate $(\beta=0.37)$ and the intake of cholesterol $(\beta=0.34)$. The low magnitude of these dietary intake tracking coefficients may also well explain the lack of findings between adolescent dietary intake and adult cardiovascular health status indicators.

The fact that both dietary intake and physical activity show low tracking contains an important public health message. It makes defining a target population for healthy lifestyle interventions difficult since there seems to be no continuity of a particular lifestyle over long periods of time. It therefore means that preventive lifestyle campaigns should target the entire population rather than predefined subsets of the population. It also means that for instance there is no guarantee that a physically active (or inversely inactive) lifestyle at a young age will automatically lead to a physically active (or again inversely inactive) lifestyle at an older age.

Regarding the relationship between adolescent physical activity and adult BMD the picture is somewhat different. Here significant relationships were found between adolescent lifestyle and adult health status. Taking the results of the Groothausen et al. ${ }^{25}$ study and the Welten et al. ${ }^{24}$ study into account, it can be concluded that 1 . in males 'energetic' adolescent physical activity contributed significantly to adult BMD; 2. both in males and females, when taking the entire longitudinal period into account, PSPA was a relatively more important predictor of adult BMD than 'energetic' physical activity and; 3. calcium intake during adolescence was not a significant predictor of bone health status measured at age 27 , both in males and females. The fact that here, in contrast to the cardiovascular findings, in males adolescent 'energetic' physical activity was related to adult bone health status may well be explained by the fact that bone mineral accrual of the lumbar spine attains approximately $60 \%$ of the maximal adult bone mineral content during the years of growth spurt, as shown by Bailey et al. ${ }^{31}$. In order to maximise bone mineral accrual during this adolescent period of growth physical activity is a prerequisite. During the adolescent years the absolute average level of 'energetic' physical activity in the male population of the AGHLS was quite high; approximately 4000 METs/ week $^{29}$ and will therefore have contributed to adult BMD. In females during adolescence this absolute average level of 'energetic' physical activity was lower; approximately $3300 \mathrm{METs} /$ week. When the entire period of exposure was taken into account PSPA was more potent predictor of adult BMD than 'energetic' physical activity. This can be explained by the fact that over the 15 years of exposure a dramatic fall of the absolute level of physical activity occurred (42\% and $17 \%$ in the males and females respectively) and by the fact that at the same time the relative contribution of more intense forms of physical activity, predominantly including PSPA, to the total amount of physical activity increased $^{32}$. The fact that in our study calcium intake during adolescence was not of influence on adult bone health was most likely because of the high levels of daily calcium intake in the Dutch population; in this cohort of relatively healthy young people even in the lowest quartile of calcium intake, the mean calcium intake was already close to the recommended daily value $^{24}$.

A second purpose of this paper was to review findings of the AGHLS regarding the longitudinal relationships between the development of physical activity and nutrition, and the development cardiovascular health status indicators. In other words: to analyse the relationship between changes in lifestyle and changes in cardiovascular health status indicators, over a 15 year period. With regard to physical activity the studies reviewed were fairly consistent: a significant positive longitudinal relationship was observed with HDL and significant negative longitudinal relationships were observed with the subscapular skinfold, and with body fat mass (SSF). These findings are in accordance with what one might expect and with what is reported in the literature ${ }^{26}$. These findings signify the importance of physical activity in preventing cardiovascular disease, since both a high level of serum HDL and low body fat mass are associated with a reduced risk for cardiovascular disease.

With regard to nutritional factors a positive longitudinal relationship was found between TC and, cholesterol intake, SFA intake and the Keys-score. A negative longitudinal relationship was found between TC and, PUFA and total energy intake. For HDL a positive longitudinal relationship was found with SFA intake. For none of the other biological health status indicators for cardiovasular disease significant longitudinal relationships were found with the dietary intake factors. The observed negative association between TC and PUFA intake and the observed positive associations between TC and, SFA and cholesterol intake are in accordance with the literature ${ }^{4}$. The Netherlands Nutrition Council has set guidelines (recommended daily allowances, RDA's) for a healthy diet. In general the daily food intake of our co-hort did not meet these healthy diet guidelines in any of the years of measurement. For seven macronutrients (total protein, total fat, SFA, PUFA, cholesterol, total carbohydrates and polysaccharides intake) the deviation of the mean intake from the RDA's was calculated. For all these macronutrients this deviation was significant, unfavourable and biologically meaningful ${ }^{4}$. This deviation from the RDA's may explain the observed 'unhealthy' longitudinal relationship between TC and some of the nutrition intake variables and stresses the need for continued efforts to advocate a healthy diet. 
The negative association between TC and total energy intake and the positive association between HDL and SFA intake are counter-intuitive and not in accordance with the literature. However, we have no sound explanation for these findings.

\section{Conclusions}

This review of 15 years of follow-up on the longitudinal relationships between lifestyle and cardiovascular and bone health status indicators showed that:

1. adolescent physical activity was not related to most of the indicators of adult cardiovascular health status, with the exception of a positive relationship with the WHR in females;

2. in males 'energetic' adolescent physical activity contributed significantly to adult BMD;

3. both in males and females, when taking the entire longitudinal period into account, PSPA was a relatively more important predictor of adult BMD than 'energetic' physical activity;

4. calcium intake during adolescence was not a significant predictor of bone health status measured at age 27 , both in males and females;

5. a consistent significant positive longitudinal relationship was found between physical activity and HDL and significant negative longitudinal relationships were found with the subscapular skinfold and with body fat mass (SSF);

6. a positive longitudinal relationship was found between TC and, cholesterol intake, SFA intake and the Keys-score; a negative longitudinal relationship was found between TC and, PUFA and total energy intake; for HDL a positive longitudinal relationship was found with SFA intake;

7. low tracking was found for physical (in-)activity and dietary intake variables.

\section{Acknowledgements}

This study was granted by the Dutch Heart Foundation (grant no. 76051-79051 and 90-312), the Dutch Prevention Fund (grant no. 28-189a, 28-1106 and 281106-1), the Dutch Ministry of Well Being and Public Health (grant no. 90-170), the Dairy Foundation on Nutrition and Health and NOC/NSF.

\section{References}

1 Pate RR, Pratt M, Blair SN, Haskell WL, Macera CA, Bouchard C, Bachner D, Ettinger W, Heath GW, King AC, Krisha A, Leon AS, Marcus BH, Marcus J, Paffenbanger RS, Patrick K, Pollock ML, Rippe JM, Sallis J, Wilmore JH. Physical activity and public health: A recommendation from the Centers for Disease Control and Prevention and the American College of Sports Medicine. J. Am. Med. Assoc. 1995; 273: 402-7.
2 Prentice AM, Jebb SA. Obesity in Britain: gluttony or sloth? Brit. Med. J. 1995; 311 (7002): 437-9.

3 Anonymus. Obesity. Preventing and managing the global epidemic. Geneva, WHO/NUT/NCD/98.1, 1998.

4 Post GB, Kemper HCG, Twisk J, van Mechelen W. The association between dietary patterns and cardio vascular disease risk indicators in healthy youngsters: Results covering fifteen years of longitudinal development. Eur. J. Clin. Nutr. 1997; 51: 387-93.

5 Riddoch C, Boreham CAG. Physical activity, fitness and children's health: current concepts. In: Armstrong N, van Mechelen W, eds. The Oxford Textbook of pediatric sports exercise science and medicine. Oxford University Press, Oxford, UK (in press).

6 Twisk JWR, Kemper HCG, Mellenbergh GJ, van Mechelen W, Post GB. Relation between the longitudinal development of lipoprotein levels and "life style" parameters during adolescence and young adulthood. An. Epid. 1996; 6 (3): 246-56.

7 Post GB. Nutrition in adolescence, a longitudinal study in dietary patterns from teenage to adult. Thesis Agricultural University Wageningen, Haarlem: de Vrieseborch, 1989.

8 NEVO-table. De Nederlandse Voedingsmiddelen Tabel (in Dutch), 1991

9 Verschuur R. Daily physical activity and health. Longitudinal changes during the teenage period. Thesis Universiteit van Amsterdam, Haarlem: de Vrieseborch, 1987.

10 Huang TC, Chen CP, Wefler V, Raftery A. A stable reagent for the Lieberman Buchard reaction: application to rapid serum cholesterol determination. Analyt.l Chem. 1961; 33: 1405-7.

11 Abell LL, Levy BB, Brody BB, Kendal FE. Simplified method for the estimation of total cholesterol in serum and demonstration of its specificity. J. Biol. Chem. 1952; 195 357-66.

12 Burstein M, Samaille J. Sur une dosage rapide du cholesterol lié aux-alpha-et aux bétalipoprotéines du serum. Clinica Chim Acta 1960; 5: 609-11.

13 Weiner JS, Lourie JA. Human Biology. A guide to field methods. IBP Handbook No. 9, Oxford: Blackwell, 1968.

14 Kemper HCG, Verschuur R. Maximal aerobic power in 13and 14-year old teenagers in relation to biological age. Int.J. Sports Med. 1981; 2: 97-100.

15 Zeger SL, Liang KY. Longitudinal data analysis for discrete and continuous outcomes. Biometrics 1986; 42: 121-130.

16 Gebski V, Leung O, McNeil D, Lunn D. SPIDA User manual. version 6. Australia, NSW: Macquarie University, 1992.

17 Twisk JWR, van Mechelen W, Kemper HCG, Post GB. The relation between "long-term exposure" to lifestyle during adolescence and young adulthood and risk factors for cardiovasular disease at adult age. J. Adolescent Health 1997; 20: 309-19.

18 van Lenthe FJ, van Mechelen W, Kemper HC, Post GB. Behavioral variables and the development of a central pattern of body fat from adolescence into adulthood in normal-weight whites: the Amsterdam Growth and Health Study. Am. J. of Clin. Nutr. 1998; 67: 846-52.

19 Twisk JWR, Kemper HCG, Mellenbergh GJ, van Mechelen W. Relation between the longitudinal development of lipoprotein levels and lifestyle parameters during adolescence and young adulthood. Ann. of Epid. 1996; 6: 246-56.

20 Twisk JWR, Kemper HCG, van Mechelen W, Post GB, van Lenthe FJ. Body fatness: longitudinal relationship of body mass index and the sum of skinfolds with other risk factors for coronary heart disease. Int. J. Obesity 1998; 22: 915-22.

21 Kemper HCG, Post GB, Twisk JWR, van Mechelen W Lifestyle and obesity in adolescence and young adulthood: results from the Amsterdam Growth and Health Longitudinal Study (AGAHLS). Int. J. Obesity 1998; 23 (suppl.3): S34-S40.

22 van Mechelen W, Twisk JWR, van Lenthe FJ, Post GB, Snel J Kemper HCG. Longitudinal relationships between resting 
heart rate and biological risk factors for cardiovascular disease: the Amsterdam Growth and Health Study. J. Sport Sci. 1998; 16: S17-S23.

23 Keys A, Anderson JT, Grande F. Serum cholesterol response to changes in diet, I-IV. Metabolism 1965; 14: 747-87.

24 Welten DC, Kemper HCG, Post GB, van Mechelen W, Twisk JWR, Lips P, Teule GJ. Weight bearing activity during youth is a more important factor for peak bone mass than calcium intake. J. Bone Min. Res. 1994; 9 (7): 1089-96.

25 Groothausen J, Siemer H, Kemper HCG, Twisk J, Welten D. Influence of peak strain on lumbar bone mineral density: an analysis of 15-year physical activity in young males and females. Ped. Ex. Sci. 1997; 9: 159-73.

26 US Department of Health and Human Services. Physical activity and health; a report of the surgeon general. United States, Atlanta, GA: National Center for Disease Control and Prevention, 1996.

27 American College of Sports Medicine. Position stand: the recommended quantity and quality of exercise for developing and maintaining cardiorespiratory and muscular fitness in healthy adults. Med. Sci. Sports Ex. 1990; 22: 265-74.
28 Twisk JWR, van Mechelen W, Kemper HCG, Post GB Tracking of risk factors for coronary heart disease over a 14 year period: a comparison between lifestyle and biological risk factors with data from the Amsterdam Growth and Health Study. American J. Epid. 1997; 145 (10): 888-98

29 van Mechelen W, Kemper HCG. Habitual physical activity in longitudinal perspective. In: The Amsterdam Growth Study. A Longitudinal Analysis of Health, Fitness and Lifestyle. Champaign, Ill. Human Kinetics Publ., HK Sport Science Monograph Series Vol. 6. 1995: 135-59.

30 Malina RM. Tracking of physical activity and physical fitness across the lifespan. Res. Q. Ex. Sport 1996; 67 (Suppl to No. 3): S1-S10.

31 Bailey DA. The Saskatchewan pediatric bone mineral accrual study: bone mineral acquisation during the growing years. Int. J. Sports Med. 1997; 18 (Suppl.3): S191-94.

32 van Mechelen W, Twisk JWR, Post GB, Snel J, Kemper HCG Habitual physical activity of young Dutch males and females: 15 years of follow-up in the Amsterdam Growth and Health Study. Med. Sci. Sports Ex. (in press). 